\title{
Safety in surgery: the checklist
}

F or surgeons, a safe surgical procedure is intrinsic to the very nature of the care we provide. We intend every operation to improve the health and well-being of our patients, and we know that a beneficial outcome depends on the proper performance of each individual operative step. We learned during our surgical training that postoperative problems can almost always be traced back to what happened during the operation.

Improving safety in the operating room (OR), we now know, requires more than simply performing the operation well. The systematic study of errors in high-risk environments-for example, the OR and the aviation industry-has taught us that errors most often result from flaws in the system, rather than from personal neglect or failure. Improved safety requires more than identifying poor performance and inspiring individual surgeons to pay more attention to detail.

One special case of errors in the OR, the retained foreign body (RFB), has received particular attention of late in the medical and lay press. Regulatory bodies have begun to view RFBs as events that should never happen, and some insurers-including Medicare in the US-now refuse to pay for the costs of care resulting from these 'preventable' events. In this context, studies have begun to shed light on the risk factors for and incidence of RFBs (Gawande, A. A. et al. N. Engl. J. Med. 348, 229-235 [2003]; Egorova, N. et al. Ann. Surg. 247, 13-18 [2008]; Cima, R. et al. J. Am. Coll. Surg. 207, 80-87 [2008]). Risk factors for RFBs varied among these studies, but are largely intuitive: emergency procedures and those of long duration, major unplanned changes in procedure, multiple changes in nursing staff, and high BMI are all implicated. Yet, RFBs also occur in cases with none of these risk factors. Clearly, better means of preventing these events are required.

The major tool used to reduce the incidence of RFBs is a count of sponges, needles and instruments at the beginning and end of each operation. One large study (Egorova et al.) reported discrepant counts in $0.7 \%$ of operations. In these cases, a missing item was found in patients only $1.6 \%$ of the time. Nevertheless, a discrepant count was associated with $77 \%$ of all RFBs, and conferred a more than 100-fold increase in the risk for a RFB. By contrast, several studies report that up to half of all RFBs occur in cases with correct counts. Radiographs taken in the OR for discrepant counts detect some, but not all, RFBs, and some institutions now routinely take a high resolution radiograph of the operated body cavity before a patient is moved to the recovery room. Such films can detect unsuspected RFBs, even in patients with correct counts, but the logistics and cost of routine radiographs are likely to delay implementation until studies confirm their benefits. Additionally, new technology to automate sponge counts and detect sponges by radiofrequency tagging might substantially reduce the incidence of retained sponges.

In this issue of Nature Reviews Urology, Birkmeyer and Miller (page 245) discuss a remarkable study from the Safety Saves Lives Study Group, which documents the value of a simple safety checklist in reducing perioperative morbidity and mortality (Haynes, A. B. et al. N. Engl. J. Med. 360, 491-499 [2009]). The group evaluated a checklist, developed from the 2008 WHO Guidelines for Safe Surgery, which calls the surgical team-surgeon, anesthetist, and nurse - to 'sign in' before the induction of anesthesia, to 'time out' before the incision, and to 'sign out' at the end of the procedure. In hospitals around the world, urban and rural, from rich and poor areas, introduction of the checklist reduced major inpatient complications and mortality by nearly half within 1 year. The effect of the checklist on actual practice was assessed by monitoring compliance with six key safety processes: objective airway evaluation; pulse oximetry; adequate intravenous access; antibiotic prophylaxis (when indicated); confirmation of identity and operative site; and sponge count. The rate of compliance with all six practices increased from $34 \%$ before the checklist was introduced to $57 \%$ afterward. More than any other study, this report documents the power of teamwork in reducing surgical complications.

Spurred on by these and other high-quality studies, surgeons worldwide have begun to realize that 'safety in the OR' depends on more than just a well-performed procedure. Verification of patient identity, marking the incision site, and a 'time out' before incision are required by the American Joint Commission before every operation. Whether the incidence of preventable errors such as RFBs can be further reduced solely through enhanced teamwork, or whether it will require better use of technology (for example, high-resolution imaging in the OR, or sensors to detect RFBs), requires further study. In the meantime, surgeons should embrace the power of teamwork, open communication, and surgical checklists as proven, low-cost, high-yield ways to further improve patient safety.

doi:10.1038/nrurol.2009.76
4 errors most often result from flaws in the system, rather than from personal neglect or failure $y$

Peter T Scardino is the Editor-in-Chief of Nature Reviews Urology.

Competing interests The author declared no competing interests. 